\title{
ALGORITMO DE BUSCA DISPERSA APLICADO AO PROBLEMA CLÁSSICO DE ROTEAMENTO DE VEÍCULOS
}

\author{
Nélida Gladys Maquera Sosa \\ Programa de Engenharia de Produção / COPPE \\ Universidade Federal do Rio de Janeiro (UFRJ) \\ nelidagladys@yahoo.com
}

\section{Roberto Diéguez Galvão*}

Programa de Engenharia de Produção / COPPE Universidade Federal do Rio de Janeiro (UFRJ) galvao@pep.ufrj.br

\section{Dan Abensur Gandelman}

Programa de Engenharia de Produção / COPPE Universidade Federal do Rio de Janeiro (UFRJ) dangandelman@hotmail.com

\footnotetext{
* Corresponding author / autor para quem as correspondências devem ser encaminhadas

Recebido em 06/2006; aceito em 03/2007 após 1 revisão Received June 2006; accepted March 2007 after one revision
}

\section{Resumo}

Neste artigo apresentamos heurísticas usando o conceito da meta-heurística Busca Dispersa (BD), desenvolvidas para a solução do Problema de Roteamento de Veículos (PRV) Clássico, detalhando cada uma de suas etapas básicas quando aplicadas ao problema em questão. A Busca Dispersa é um método evolutivo que combina soluções com a finalidade de criar novas soluções de melhor qualidade; ainda que apresente similaridades com os algoritmos genéticos difere dos mesmos em princípios fundamentais. Um aspecto importante da BD é formar soluções com alta qualidade para dirigir a busca a regiões promissoras. Experimentos computacionais foram realizados em quatro conjuntos de dados disponíveis na literatura. Os resultados mostram que a BD é robusta e competitiva em termos de qualidade das soluções obtidas e tempo computacional para o PRV Clássico, para os conjuntos de dados testados.

Palavras-chave: busca dispersa; problema de roteamento de veículos; meta-heurísticas.

\begin{abstract}
In this paper we present a Scatter Search algorithm designed for the solution of the Classical Vehicle Routing Problem, giving details of each of its basic phases when applied to the routing problem. Scatter Search is an evolutionary meta-heuristic that combines solutions with the objective of obtaining new solutions of higher quality; even though there are similarities between Scatter Search and Genetic Algorithms, the two methods differ in basic principles. An important aspect of Scatter Search is to construct high quality solutions in order to direct the search to promising regions. Computational experiments were conducted using four data sets available in the literature. The results show that Scatter Search is robust and competitive in terms of both the quality of solutions and computational times, for the VRP data sets we used.
\end{abstract}

Keywords: scatter search; vehicle routing problem; meta-heuristics. 


\section{Introdução}

A importância de sistemas de distribuição torna-se evidente quando se considera o impacto dos respectivos custos na operação das empresas. Existe uma variedade de problemas de decisão nessa área, em diferentes níveis: estratégico, tático e operacional. Decisões sobre a localização de facilidades (fábricas, depósitos, entre outras) são consideradas estratégicas; decisões que determinam por exemplo o tamanho e a composição da frota de veículos de uma empresa podem ser consideradas de nível estratégico/tático; a nível operacional temos por exemplo decisões sobre o roteamento e/ou sequenciamento de frotas de veículos.

O Problema de Roteamento de Veículos (PRV) é um nome genérico dado a uma classe de problemas de distribuição. Um caso particular do problema geral constitui a chamada versão clássica do PRV e pode ser definida da seguinte maneira. Seja $G=(U, A)$ um grafo (direcionado ou não) onde $U=\left\{u_{0}, u_{1}, \ldots, u_{n}\right\}$ é o conjunto de vértices (clientes) $\mathrm{e}$ $A=\left\{\left(u_{i}, u_{j}\right): u_{i}, u_{j} \in U, i \neq j\right\}$ é o conjunto de arcos. O vértice $u_{0}$ representa o depósito de uma frota homogênea de veículos com capacidade $Q$ por veículo. Cada cliente tem uma demanda $q_{i}$ e requer um tempo de serviço $s_{i}$. Uma matriz de custos de viagem $\mathrm{C}=\left[c_{i j}\right]$ é definida em A. O número de veículos é pré-determinado ( $m$ veículos) ou é considerado uma variável de decisão. O PRV clássico (com restrições de capacidade e distância máxima) consiste em designar um conjunto de $m$ rotas de entrega e/ou coleta tal que: i) o custo total do conjunto de rotas percorrido pela frota seja minimizado; ii) cada rota se inicie e finalize no depósito; iii) cada cliente tenha sua demanda suprida exatamente por um veículo; iv) a carga total de cada veículo não exceda sua capacidade $Q$; e v) o tempo total necessário para completar qualquer rota não exceda um limite pré-especificado $T$, que inclui tempos de viagem entre clientes e tempos de serviço em cada cliente.

O PRV é um problema NP-difícil (Lenstra \& Rinnooy Kan, 1981). Devido à sua complexidade computacional, métodos exatos de solução são inviáveis para instâncias de grande porte, o que nos remete à utilização de métodos heurísticos/meta-heurísticos. Existe um grande número de artigos que tratam do problema clássico descrito no parágrafo acima, usando diversas heurísticas e meta-heurísticas, com predominância das meta-heurísticas, mais recentes e apresentando resultados de melhor qualidade. Não é nosso objetivo fazer uma revisão detalhada da bibliografia existente sobre o assunto; uma revisão detalhada (mas evidentemente não atualizada) sobre o PRV pode ser encontrada em Laporte et alii (2000). Alguns artigos recentes relacionados ao tema são, por exemplo, Cordeau et alii (2002), Baker \& Ayechew (2003), Vigo \& Toth (2003), Wassan (2006). Toth \& Vigo (2002) escreveram um importante livro sobre o PRV, englobando tanto o problema clássico como variantes do mesmo.

Recentemente a Busca Dispersa (BD), uma nova metodologia com base em populações, tem demonstrado ser muito efetiva na solução de problemas de otimização discreta. Apesar de a Busca Dispersa apresentar algumas semelhanças com os Algoritmos Genéticos (AG), ela difere dos mesmos em princípios fundamentais, tais como o uso de estratégias determinísticas ao invés de estratégias aleatórias. Um aspecto importante da BD é a relação existente entre a capacidade do método de dirigir a busca a regiões promissoras e sua eficiência na exploração dessas regiões.

A BD proporciona o uso de estratégias flexíveis, que permitem o desenvolvimento de diferentes algoritmos com distintos graus de complexidade para as diferentes etapas da busca. Os conceitos e princípios fundamentais do método foram propostos na década de 70 por Glover 
(1977), com base em estratégias para combinar regras de decisão e restrições. Os trabalhos de Glover (1997), Laguna \& Martí (2003) e Martí et alii (2006) são referências importantes sobre a BD. A Busca Dispersa tem por base o principio de que, dadas duas soluções, pode-se obter novas soluções mediante a combinação delas, de modo a melhorar as que as originaram.

Neste artigo propomos um algoritmo com base na meta-heurística Busca Dispersa para o PRV Clássico, testando-o para diferentes conjuntos de dados. O texto está organizado da seguinte forma. Na Seção 2 apresentamos uma descrição básica da BD. Na Seção 3 descrevemos detalhadamente o algoritmo de BD que propomos para o PRV Clássico. Testes computacionais realizados com diferentes conjuntos de dados são mostrados na Seção 4. Por último, na Seção 5, fazemos algumas considerações finais sobre o trabalho.

\section{A Meta-heurística Busca Dispersa}

A Busca Dispersa (Scatter Search em inglês) é um método evolutivo (baseado em populações) que é muito efetivo na solução de diversos problemas de otimização discreta, por exemplo na solução do problema de ordenação linear, ver Campos et alii (2001), na otimização global de funções multimodais, ver Laguna \& Martí (2000) e na solução de problemas de roteamento de veículos com janelas de tempo, ver Russel \& Chiang (2006). Essa meta-heurística combina soluções pertencentes a um conjunto denominado conjunto de referência (Refset), com o intuito de capturar informação não contida nas soluções originais. O Refset guarda "boas" soluções encontradas durante o processo de busca. Cabe destacar que o significado de "boa" não se restringe apenas à qualidade da solução, mas também a sua diversidade em relação a outras soluções deste conjunto. A BD é composta basicamente por 5 etapas (métodos), que descrevemos a seguir de forma resumida. Posteriormente explicaremos detalhadamente cada etapa no contexto de uma meta-heurística desenvolvida visando a solução do PRV Clássico. Apresentamos inicialmente a nomenclatura que será utilizada neste trabalho.

$P \quad$ Conjunto de soluções obtidas com o método gerador de soluções iniciais;

PSize Tamanho da população $P$ de soluções iniciais (i.e., $|P|=P$ size);

Refset Conjunto de Referência;

Pool Conjunto de soluções geradas pelo método da combinação;

$b \quad$ Tamanho do conjunto de referência (i.e., $|\operatorname{RefSet}|=b$ );

$b_{1} \quad$ Tamanho do subconjunto do Refset correspondente a soluções de qualidade;

$b_{2} \quad$ Tamanho do subconjunto do Refset correspondente a soluções com diversidade;

$x^{i} \quad$ A $i^{e ́ s i m a}$ solução do conjunto de referência. $x^{1}$ é a melhor solução em Refset e $x^{b}$ a pior;

$d(x, y)$ Distância entre a solução $x$ e a solução $y$.

As etapas da BD são as seguintes:

1. Etapa Geradora de Soluções Iniciais: esta etapa gera um conjunto $P$ com Psize soluções, de onde extraímos um subconjunto que se denomina conjunto de referência Refset.

2. Etapa de Melhoria: consiste de uma busca local para melhorar as Psize soluções inicialmente encontradas. Se a solução que está sendo avaliada é inviável, o método tenta transformá-la em uma solução viável. Se a solução é viável, o método tenta melhorá-la.

3. Geração do Conjunto de Referência: nesta etapa é construído ou atualizado o conjunto de referência Refset. 
3.1 Construção - A partir do conjunto $P$ se extrai Refset, combinando soluções de alta qualidade com soluções com diversidade; as soluções de Refset são usadas, durante o processo de busca, para gerar novas soluções mediante a combinação de soluções contidas nesse conjunto.

3.2 Atualização - A atualização de Refset acontece quando novas soluções que atendem os requisitos para ingressar em Refset são encontradas. Assim Refset mantém seu tamanho $b=b_{1}+b_{2}$ constante, mas as soluções pertencentes ao mesmo melhoram durante o processo de busca.

Para atualizar Refset tem-se que considerar o momento em que a atualização deve ser realizada (estática ou dinâmica). A atualização se diz estática quando a mesma é realizada após testadas todas as possíveis combinações em Refset; se diz dinâmica quando imediatamente após alguma combinação a solução resultante é tentativamente inserida em Refset, sempre que atenda os requisitos necessários para ingressar no conjunto.

4. Geração de Subconjuntos: opera no conjunto de referência, consistindo em criar diferentes subconjuntos de soluções que serão utilizadas na etapa de combinação.

5. Combinação de Soluções: utiliza os subconjuntos de soluções gerados na Etapa 4, combinando as soluções em cada subconjunto com o objetivo de encontrar novas soluções. Esta etapa de combinação é um mecanismo específico para cada problema, uma vez que está diretamente relacionado com a representação da solução.

O algoritmo da Figura 1 mostra as etapas descritas por 1-5 em um esquema básico da Busca Dispersa para um problema de minimização (atualização de Refset estática).

Passo 1. Inicie com $P \leftarrow \varnothing$. Inicie a etapa geradora de soluções iniciais e construa uma solução $y$. Aplique o método de melhoria a $y$ e encontre a solução melhorada $x$. Se $x \notin P$ então adicione $x$ a $P$, caso contrário descarte $x$. Repita este passo até $|P|=P$ size.

Passo 2. Construa o conjunto de referência Refset $=\left\{x^{1}, \ldots, x^{b}\right\}$ com as $b_{1}$ melhores soluções de $P$ e as $b_{2}$ soluções de $P$ com maior diversidade em relação às soluções que estão em Refset. Ordene essas $b=b_{1}+b_{2}$ soluções de forma crescente em relação à função objetivo. Faça o operador lógico newsolutions $\leftarrow$ TRUE.

Enquanto (newsolutions $=$ TRUE.)

newsolutions $\leftarrow$ FALSE.

Passo 3. Mediante a geração de subconjuntos gere subconjuntos a partir de RefSet, agrupando por exemplo pares de soluções desse conjunto.

Enquanto (existem subconjuntos sem examinar)

Passo 4. Aplique o método de combinação às soluções de cada subconjunto gerado no Passo 3 e guarde as soluções obtidas em uma lista denominada Pool.

Passo 5. Aplique o método de melhoria a cada solução de Pool obtida no Passo 4.

Fim Enquanto

Passo 6. Caso possível atualize Refset ( $b$ melhores soluções em Refset $\cup$ Pool);

Reordene Refset.

Se Refset atualizado, newsolutions $\leftarrow$ TRUE.

Fim Enquanto

Figura 1 - Esquema Básico de um Algoritmo de Busca Dispersa.

$\mathrm{O}$ algoritmo básico de $\mathrm{BD}$ termina quando não existem novos elementos a serem combinados em Refset. 


\section{Uma Aplicação da Busca Dispersa ao PRV Clássico}

Nesta seção são apresentadas as diferentes etapas de uma aplicação da BD ao PRV clássico. Para a etapa geradora de soluções foram testadas duas heurísticas construtivas, apresentadas a seguir. São apresentados também três métodos de melhoria inter-rotas e um método de melhoria intra-rota para a etapa de melhoria da BD. As etapas de construção (atualização) de Refset, geração de subconjuntos e combinação de soluções são finalmente descritos.

A construção de Refset se inicia a partir do conjunto $P$ de Psize soluções geradas após as duas etapas iniciais da busca. As soluções de Refset são ordenadas em ordem crescente do valor da função objetivo, isto é, de acordo com sua qualidade. Seleciona-se os pares de soluções que serão combinadas e o processo de combinação de soluções de Refset é iniciado. Refset é atualizado com novas soluções que forem geradas por esse processo; a atualização de Refset pode ser estática ou dinâmica. O critério de parada para a BD é quando for atingido o valor da solução ótima da instância em consideração (quando disponível na literatura), ou quando Refset não for atualizado na última etapa da BD.

\subsection{Etapa 1: Geração de Soluções Diversas}

As heurísticas desenvolvidas para o PRV Clássico podem ser classificadas em três categorias distintas: heurísticas de inserção, heurísticas de duas fases e heurísticas de melhoria. As heurísticas de inserção constroem gradualmente uma solução pela inserção de clientes de acordo com algum critério pré-estabelecido. Nas heurísticas de duas fases o problema é decomposto em duas fases: agrupamento e roteamento. Pode ser feito inicialmente o agrupamento de clientes, seguido pelo seu roteamento; ou as duas fases podem ser invertidas (primeiro o roteamento, seguido do agrupamento). As heurísticas de melhoria podem ser de melhoria inter-rotas ou intra-rotas.

Apresentamos a seguir dois métodos para gerar as Psize soluções iniciais (população inicial) da BD: o primeiro uma adaptação da Heurística de Varredura de Gillett \& Miller (1974); o segundo com base na heurística de Beasley (1983). Os resultados produzidos por esses algoritmos são melhorados por meio de diversos procedimentos simples na etapa seguinte da BD.

\subsubsection{Algoritmo G \& M: Com base na heurística de Gillett e Miller}

Esta heurística corresponde a um algoritmo de agrupamento-roteamento. Na heurística de varredura com base no algoritmo proposto por Gillett \& Miller (1974), os agrupamentos são formados girando uma semi-reta com origem no depósito e incorporando os clientes "varridos" por dita semi-reta na mesma rota até que as restrições do PRV Clássico não sejam satisfeitas. Para este algoritmo cada cliente $i$ está dado por suas coordenadas polares $\left(\rho_{i}, \theta_{i}\right)$, em um sistema que tem o depósito como origem. Uma versão simplificada da heurística é definida pelos passos descritos a seguir.

Passo 1: Ordenar os clientes segundo o ângulo polar $\theta$ que cada cliente faz com a semireta. Se dois clientes possuem o mesmo valor de $\theta$, o cliente com menor valor de $\rho$ é selecionado. Selecione um cliente $u_{i}$ como cliente inicial e faça $i=1, k=1$; $C_{1}=\left\{u_{1}\right\}$ é o cluster de ordem 1 .

Passo 2: Se todos os clientes pertencem a algum cluster, ir ao Passo 4. Caso contrário ir ao Passo 3. 
Passo 3: Fazer $i=i+1$ e selecionar o próximo cliente $u_{i}$. Se $u_{i}$ puder ser adicionado a $C_{k}$, fazer $C_{k}=C_{k} \cup\left\{u_{i}\right\}$. Caso contrário, fazer $k=k+1$ e criar um novo agrupamento $C_{k}=\left\{u_{i}\right\}$. Ir ao Passo 2.

Passo 4: Para cada agrupamento $C_{k}$, resolver um Problema do Caixeiro Viajante.

Uma solução viável do PRV é assim construída. O processo se repete $n$ vezes (onde $n$ é o número de clientes, $n=P$ size $)$, iniciando cada execução do algoritmo por um cliente diferente.

\subsubsection{Algoritmo B: Com base na heurística de Beasley}

Heurística proposta por Beasley (1983), cuja estratégia é rotear primeiro, agrupar depois. Sem considerar as restrições do PRV, na fase de roteamento constrói-se inicialmente um "tour gigante" iniciado no depósito, que percorre todos os clientes e retorna ao depósito. A este "tour gigante" aplica-se a heurística de melhoria 2-opt, na qual duas arestas do "tour" são trocadas por outras duas não pertencentes ao mesmo, sempre que o resultado da troca diminui o comprimento do "tour" (ver 3.2.2). Na fase de agrupamento as restrições do PRV são consideradas; o "tour gigante" é dividido em um número de rotas viáveis em que os clientes são designados às rotas conforme sua seqüência no mesmo. Uma solução viável do PRV é assim construída.

\subsubsection{Comparação Computacional}

Diversas experiências computacionais foram realizadas a fim de comparar o desempenho dos algoritmos construtivos com base nas heurísticas de Gillett e Miller e Beasley. Experimentos foram realizados em dois conjuntos de dados, formados respectivamente pelas 14 instâncias descritas em Christofides et alii (1979), e pelas 27 instâncias descritas em Augerat et alii (1995). As características dessas instâncias são mostradas nas tabelas A.1 e A.2 do Apêndice, respectivamente.

A Tabela 1 abaixo mostra os resultados obtidos para as duas heurísticas de construção: sem melhoria (linha 1) e depois de aplicada a heurística de melhoria (linha 2) às instâncias. Os procedimentos de melhoria (explicados na seção 3.2) foram propostos por diferentes autores, conforme descrito por exemplo em Toth \& Vigo (2002). Todos os dados da Tabela 1 são os Desvios Percentuais Médios em Relação às melhores soluções encontradas na literatura (limite superior ou solução ótima), disponíveis em http://neo.lcc.uma.es/radi-aeb/WebVRP/. Observamos na Tabela 1 que ambas heurísticas apresentam desempenhos semelhantes antes e após o procedimento de melhoria. Optamos por trabalhar com o procedimento com base na heurística de Gillett e Miller.

Tabela 1 - Comparação das heurísticas de Construção.

\begin{tabular}{l|c|c}
\hline \multicolumn{1}{c|}{ DPRM } & Gillett e Miller & Beasley \\
\hline Sem melhoria & 19,33 & 19,15 \\
Com melhoria & 9,31 & 9,13 \\
\hline
\end{tabular}

\subsection{Etapa 2: Melhoria das Soluções}

Nesta seção apresentamos as heurísticas de melhoria utilizadas na Fase 2 de nossa heurística de $\mathrm{BD}$ para o PRV clássico. Apresentamos três movimentos inter-rotas e um movimento intra-rota. 


\subsubsection{Movimentos inter-rotas}

Apresentamos a seguir três tipos de movimentos inter-rotas: re-alocação, intercâmbio e cruzamento. Os movimentos de re-alocação e intercâmbio foram definidos em artigos descrevendo heurísticas de "primeira geração" para problemas de roteamento de veículos; ver por exemplo Ball et alii [Editors] (1995). O movimento de cruzamento está por exemplo descrito em Montané \& Galvão (2006), que o utilizaram na solução do problema de roteamento de veículos com entregas e coletas simultâneas.

\section{Re-alocação}

Consiste em remover um cliente de uma das rotas e inseri-lo em uma outra rota. Nas Figuras 2(a) e 2(b) apresentamos o movimento de re-alocação. Para este exemplo o depósito é denominado por 0 e na figura está representado por um quadrado; nestas figuras o cliente $a$ é mudado de rota; ou, se desejamos considerar a re-alocação em função de arcos, os arcos $(i, 0),(a, b) \mathrm{e}(a, d)$ são substituídos respectivamente pelos $\operatorname{arcos}(i, a),(a, 0)$ e $(b, d)$.

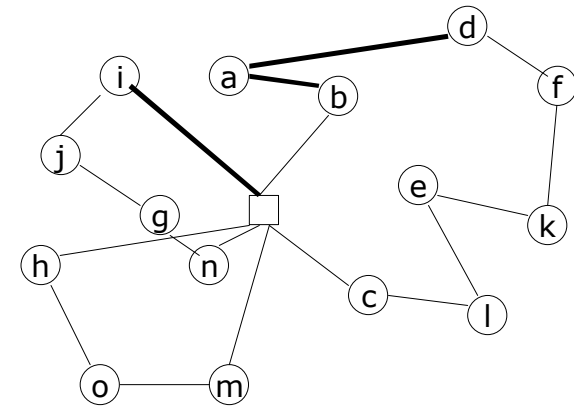

(a)

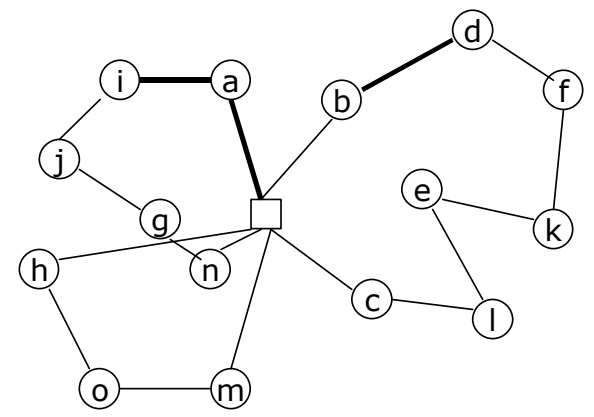

(b)

Figura 2 - Movimento de Re-alocação.

\section{Intercâmbio}

Consiste em intercambiar clientes entre duas rotas. Nas Figuras 3(a) e 3(b) os clientes $g$ e $j$ são intercambiados; ou, se desejamos expressar o intercâmbio em função de arcos, temos que os $\operatorname{arcos}(i, g),(g, 0),(h, j)$ e $(j, 0)$ são substituídos respectivamente pelos $\operatorname{arcos}(i, j),(j, 0),(h, g)$ e $(g, 0)$.

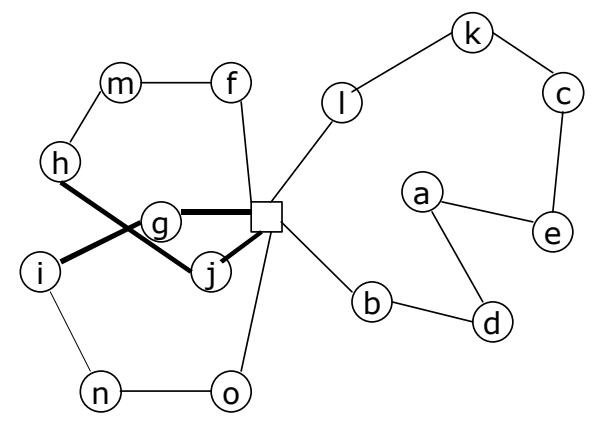

(a)

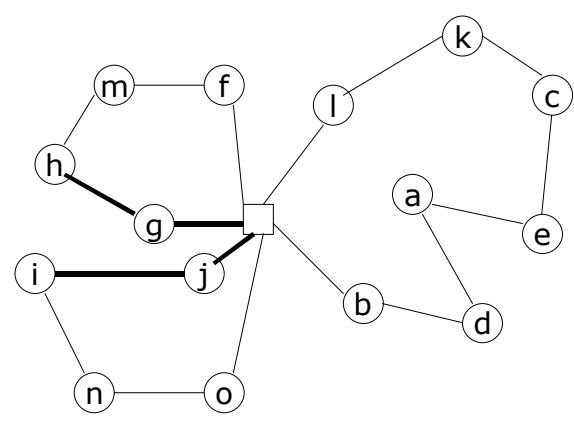

(b)

Figura 3 - Movimento de Intercâmbio. 


\section{Cruzamento}

Neste movimento seleciona-se um par de rotas. Cada rota é dividida em duas seções que são re-conectadas. Consegue-se isso removendo um arco de cada rota e substituindo-os por dois novos arcos que conectam respectivamente a seção inicial da primeira rota com a seção final da segunda rota e a seção inicial da segunda com a seção final da primeira. Na Figura 4 ilustramos este movimento para um par de rotas; a rota $\mathrm{p}_{0}-\mathrm{p}_{\mathrm{i}-1}-\mathrm{p}_{\mathrm{i}}-\mathrm{p}_{0}$ é substituída pela rota $\mathrm{p}_{0^{-}}$ $\mathrm{p}_{\mathrm{i}-1}-\mathrm{q}_{\mathrm{j}}-\mathrm{p}_{0}$ e a rota $\mathrm{q}_{0}-\mathrm{q}_{\mathrm{j}-1}-\mathrm{q}_{\mathrm{j}}-\mathrm{q}_{0}$ é substituída pela rota $\mathrm{q}_{0}-\mathrm{q}_{\mathrm{j}-1}-\mathrm{p}_{\mathrm{i}}-\mathrm{q}_{0}$.

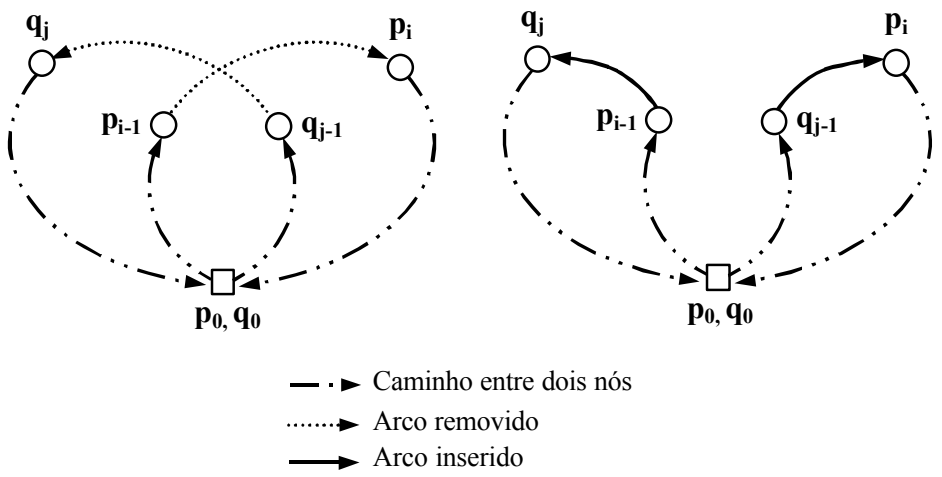

Figura 4 - Movimento de Cruzamento.

\subsubsection{Melhoria intra-rotas}

$\mathrm{Na}$ literatura são encontradas diferentes formas de realizar o movimento intra-rotas; em nosso algoritmo utilizamos o movimento 2-opt.

\section{Movimento 2-opt}

Para a vizinhança intra-rotas utilizamos a heurística 2-opt, inicialmente proposta por Lin (1965) para o problema do caixeiro viajante; os arcos $(i, o)$ e $(b, j)$ [Figura 5(a)] são trocados respectivamente pelos $\operatorname{arcos}(i, j)$ e $(b, o)$ [Figura $5(\mathrm{~b})]$.

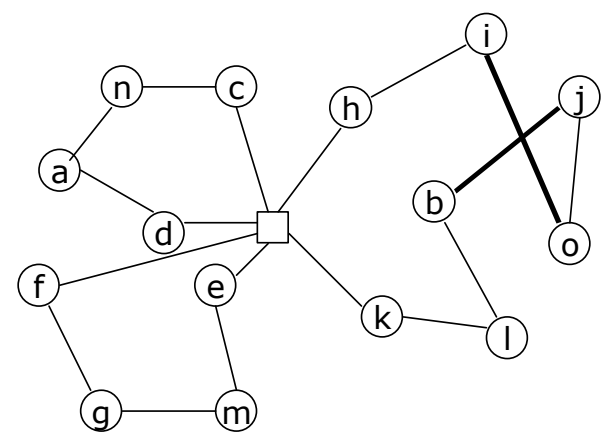

(a)

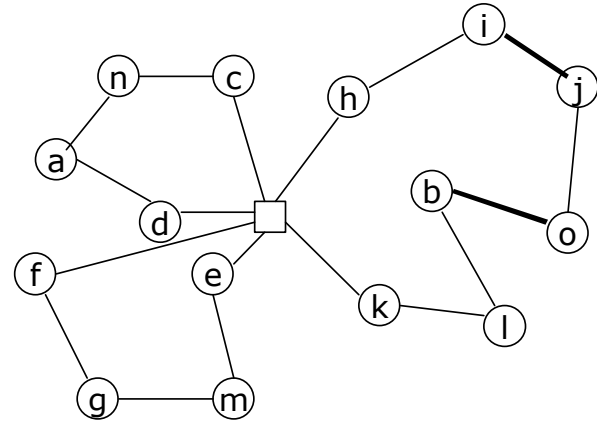

(b)

Figura 5 - Movimento 2-opt. 
Foram aplicadas três variações dos métodos de melhoria às $n$ soluções geradas com base no algoritmo de Gilett e Miller: $i$ ) melhoria quando somente realizam-se movimentos de inserção (ou re-alocação, MMI); ii) melhoria quando primeiramente realizam-se movimentos de re-alocação, seguidos de movimentos de intercâmbio e finalmente de movimentos de cruzamento, ou seja, os movimentos são realizados seqüencialmente (MMS) e iii) melhoria quando realizam-se os movimentos de re-alocação, intercâmbio e cruzamento separadamente, sendo executado, dentre o melhor movimento de cada uma das três vizinhanças, aquele que proporciona o maior ganho no valor da função objetivo (MMT). Note-se que, em qualquer um dos três casos, (i), (ii) ou (iii), escolhe-se sempre o melhor movimento de cada vizinhança analisada. O movimento 2-opt (melhoria intra-rota) é aplicado duas vezes: antes da aplicação dos movimentos inter-rotas e após a aplicação desses movimentos.

Para determinar a melhor estratégia a ser seguida na etapa de melhoria, resultados de experimentos computacionais são apresentados na Tabela 2. O mesmo conjunto das $n$ soluções foi sempre utilizado neste experimento. A Tabela 2 mostra, para as três estratégias listadas acima, o desvio percentual médio em relação às melhores soluções encontradas na literatura e o tempo computacional respectivo. Considerando esses resultados decidimos utilizar em nosso algoritmo de BD o método de melhoria MMS.

Tabela 2 - Métodos de melhoria.

\begin{tabular}{l|l|l|l}
\hline & \multicolumn{1}{|c|}{ MMI } & \multicolumn{1}{c|}{ MMS } & \multicolumn{1}{c}{ MMT } \\
\hline DPRM & 9,47 & 9,31 & 9,30 \\
Tempo (s) & 1,46 & 1,76 & 3,37 \\
\hline
\end{tabular}

\subsection{Etapa 3: Construção de Refset}

As melhores $b_{1}$ soluções do conjunto $P$ são incluídas em Refset. Outras $b_{2}$ soluções são acrescentadas a Refset tal que a diversidade de Refset seja a maior possível. É definida uma distância entre duas soluções $x$ e $y$ para determinar a diversidade entre as mesmas: $d(x, y)=\sum_{k=1}^{k=n} h_{k}$, onde $h_{k}$ é 0 se o cliente $k$ pertence à mesma rota nas soluções $x$ e $y$; é 1 caso contrário (as rotas são identificadas através de numeração das mesmas). Selecionase de $P$ a solução que maximiza a distância mínima a todas as soluções já incluídas em Refset; o processo é repetido até serem inseridas deste modo $b_{2}$ soluções nesse conjunto.

\subsection{Etapa 4: Geração de Subconjuntos}

Terminada a construção de Refset, consideramos a implementação mais simples do Método de geração de subconjuntos, que consiste em realizar unicamente combinações de soluções duas a duas. Isto é, para a geração de cada subconjunto consideramos pares de soluções de Refset que não tenham sido ainda combinadas.

\subsection{Etapa 5: Combinação de Soluções}

Após selecionar os pares de soluções a serem combinadas aplica-se o Método de Combinação de Soluções. Este método funciona da seguinte maneira: i) Identifica-se os clientes que pertencem às mesmas rotas em soluções a serem combinadas. Esses clientes são 
fixados às rotas; os clientes não fixados são desconectados das mesmas; ii) Constrói-se uma nova solução conforme explicado abaixo e iii) encontrada a nova solução, aplica-se à mesma o Método de Melhoria MMS.

Pode acontecer que após aplicado o método de combinação acima existam clientes desconectados das rotas, em cujo caso é necessária a viabilização da solução correspondente. Para isto é proposta uma heurística que considera os dados de distância e peso relativos do cliente que foi desconectado. Para cada cliente $i$ (cliente_candidato) não fixado encontra-se a rota mais próxima. Uma rota se diz próxima do cliente_candidato se a distância do último cliente da mesma ao cliente_candidato, mais a distância do cliente_candidato ao depósito, tem o menor valor possível e satisfaz às restrições do PRV Clássico. A soma dessas duas distâncias é dividida pela demanda do cliente_candidato. O cliente_candidato com o menor valor deste quociente é inserido na rota mais próxima e os dados são atualizados. Quando a inserção de um novo cliente torna a solução inviável, uma nova rota é iniciada. O processo termina quando todos os clientes forem roteados.

Na Figura 6 ilustra-se o método de combinação de um problema com 15 clientes em que as duas soluções a serem combinadas têm três veículos cada. As Figuras 6(a) e 6(b) mostram as duas soluções que serão combinadas. Na Figura 6(c) clientes comuns das soluções 1 e 2 são identificados e fixados nas rotas respectivas; a obtenção da nova solução é feita de modo que sejam formadas rotas segundo o algoritmo descrito acima, conforme mostramos na Figura 6(d).

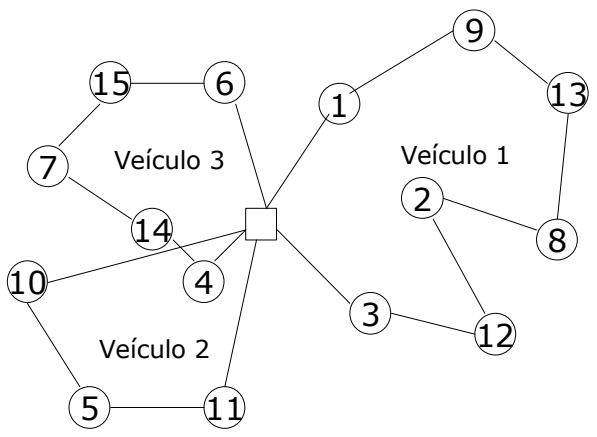

(a)

(9)

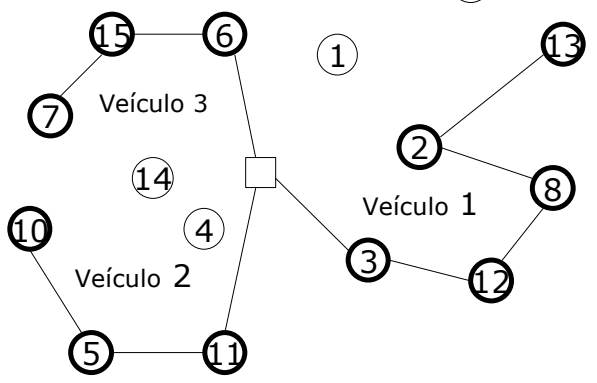

(c)

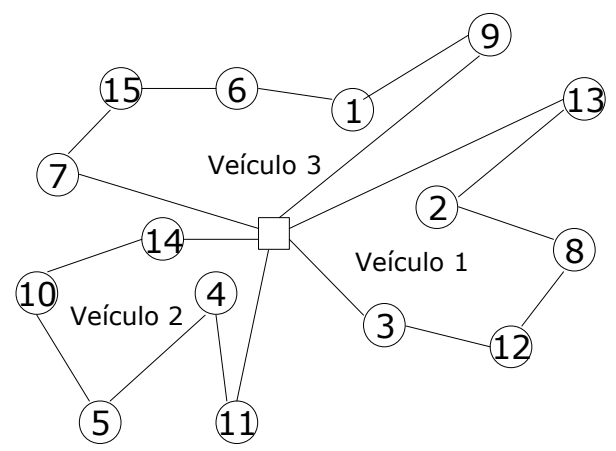

(b)

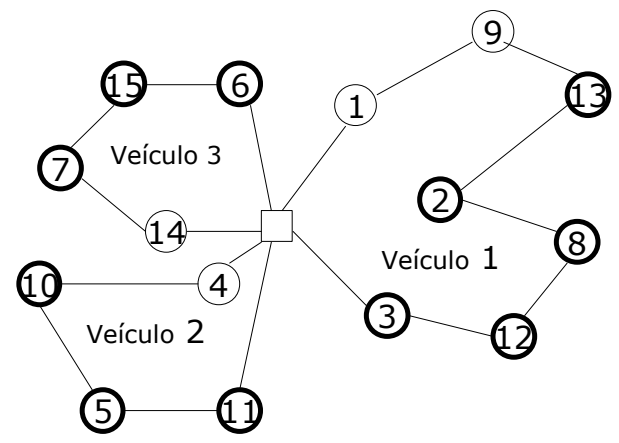

(d)

Figura 6 - (a) Solução 1; (b) Solução 2; (c) Clientes comuns; (d) Solução resultante. 


\subsection{Atualização do Conjunto de Referência}

Se a solução encontrada pelo método de combinação for de qualidade, esta pode imediatamente substituir a pior solução em Refset (atualização dinâmica) ou ser armazenada em uma lista denominada Pool (para que futuramente seja efetuada uma atualização estática). No caso de uma atualização estática Refset consistirá das $b$ melhores soluções armazenadas em Refset $\cup$ Pool. A estratégia de atualização dinâmica modifica Refset rapidamente e usualmente produz soluções em menor tempo computacional, enquanto a estratégia de atualização estática usualmente produz melhores soluções, mas em mais tempo computacional.

A Tabela 3 mostra resultados obtidos com ambas as estratégias nas instâncias de Christofides et alii (1979) e Augerat et alii (1995) para diferentes valores de $b_{1}$ e $b_{2}$. Pode-se observar na Tabela que a atualização estática sempre tem um melhor desempenho em termos de desvio percentual relativo médio (DPRM), enquanto que o tempo computacional (Tempo) em que é encontrada a melhor solução durante o processo de busca é maior na atualização estática que na atualização dinâmica. Decidimos implementar o algoritmo de BD para o PRV Clássico utilizando a atualização estática.

Tabela 3 - Atualização Estática versus Atualização Dinâmica.

\begin{tabular}{|c|c|c|c|c|c|c|}
\hline Tamanho $R$ & set (BD_ $b_{1} b_{2}$ ) & BD_5_5 & BD_2_8 & BD_3_7 & BD_7_3 & BD_10_10 \\
\hline Atualização I & stática & & & & & \\
\hline Christofides & DPRM & 0,86 & 0,98 & 1,09 & 1,08 & 0,78 \\
\hline & Tempo (s) & 66,61 & 87,53 & 88,79 & 72,20 & 123,20 \\
\hline Augerat & DPRM & 0,45 & 0,37 & 0,37 & 0,38 & 0,38 \\
\hline & Tempo (s) & 12,93 & 2,86 & 2,84 & 3,09 & 1,22 \\
\hline Atualização I & inâmica & & & & & \\
\hline Christofides & DPRM & 2,15 & 1,92 & 1,46 & 2,33 & 1,44 \\
\hline & Tempo (s) & 40,91 & 29,60 & 38,55 & 32,50 & 62,52 \\
\hline Augerat & DPRM & 0,54 & 0,62 & 0,59 & 0,56 & 0,44 \\
\hline & Tempo (s) & 2,61 & 2,50 & 2,40 & 2,25 & 1,11 \\
\hline
\end{tabular}

Se na atualização de Refset novas soluções são introduzidas, ativa-se novamente o método da combinação de soluções. O critério de parada para a $\mathrm{BD}$ acontece quando foi atingido o valor ótimo da instância respectiva (quando disponível na literatura), ou quando Refset não foi modificado, isto é, quando não foi atualizado na última etapa da BD.

\section{Resultados Computacionais}

Para avaliar o desempenho do algoritmo de BD proposto na seção anterior efetuamos testes computacionais com quatro conjuntos de dados disponíveis na literatura, apresentados nas Tabelas A.1 a A.4 do Apêndice: o Conjunto I, formado pelas 14 instâncias descritas em Christofides et alii (1979); o Conjunto II, formado por 27 instâncias descritas em Augerat et alii (1995); o Conjunto III, formado por 11 instâncias descritas em Christofides \& Eilon (1969) e o Conjunto IV, formado por 3 instâncias descritas em Fisher (1994). Nas instâncias do Conjunto I o número de clientes varia entre 50 e 199; no Conjunto II, entre 31 e 79; no Conjunto III, entre 21 e 100; e no Conjunto IV, entre 44 e 134 clientes. 
Com a finalidade de permitir uma comparação apropriada, cabe esclarecer que a distância entre pares de vértices (clientes) é dada pela distância Euclidiana (sem nenhum arredondamento) para os Conjuntos I e IV, enquanto que para as instâncias dos Conjuntos II e III à distância entre pares de vértices é acrescentado o valor de 0.5 , sendo então o valor obtido dessa soma arredondado. Além disso, conforme explicam Martinhon et alii (2004), o número de veículos para estas instâncias é um dado de entrada quando os problemas são resolvidos por meio de algoritmos exatos, mas pode ser uma variável de decisão no caso de heurísticas/ meta-heurísticas.

Os códigos para as diferentes implementações da BD foram programados na linguagem $\mathrm{C}$, em um microcomputador PC com processador Pentium 4 de $2.26 \mathrm{GHz}$. Com o objetivo de avaliar o grau de proximidade entre as soluções encontradas nas diferentes implementações da $\mathrm{BD}$ em relação às melhores soluções encontradas na literatura, foi definida uma medida de desvio. Ela é dada pelo desvio percentual relativo (DPR), DPR $=100 *((B D-F b e s t) / F b e s t)$, onde $B D$ é o valor da função objetivo encontrado pela Busca Dispersa e Fbest é a melhor solução disponível na literatura para um dado problema-teste.

Na Tabela 4 mostramos os resultados computacionais obtidos pelo algoritmo de Busca Dispersa com diferentes valores de tamanho para Refset. A Tabela 4 mostra os resultados para diferentes estratégias na geração de Refset, utilizando dois tamanhos para o mesmo: 10 e 20 soluções. Várias combinações para ambos os tamanhos foram testadas. Os valores de $b_{1}$ e $b_{2}$ representam o número de soluções escolhidas por qualidade e diversidade, respectivamente. Para cada conjunto de instâncias a Tabela 4 mostra o desvio percentual relativo médio (DPRM) em relação às melhores soluções disponíveis na literatura (ver http://neo.lcc.uma.es/radi-aeb/WebVRP/); o tempo computacional (T) da BD em segundos; o tempo necessário para encontrar a melhor solução da BD (TE), em segundos; e o tempo computacional em segundos (TMD) para construir as soluções iniciais da BD. Todos os tempos mostrados na Tabela 4 são tempos médios, para o número de instâncias de cada conjunto.

Tabela 4 - Resultados Computacionais da Busca Dispersa: Atualização Estática.

\begin{tabular}{l|l|r|r|r|r|r}
\hline \multicolumn{1}{c}{ Instâncias } & & BD_5_5 & BD_2_8 & BD_3_7 & BD_7_3 & BD_10_10 \\
\hline Conjunto I & DPRM & 0,86 & 0,98 & 1,09 & 1,08 & 0,78 \\
(14 inst.) & T (s) & 127,55 & 128 & 124,46 & 142,91 & 213,94 \\
& TE(s) & 66,61 & 87,53 & 88,79 & 72,20 & 123,20 \\
& TMD(s) & 33,32 & 32,35 & 35,30 & 34,46 & 47,83 \\
Conjunto II & DPRM & 0,45 & 0,37 & 0,37 & 0,38 & 0,38 \\
(27 inst.) & T (s) & 12,93 & 2,86 & 2,84 & 3,09 & 3,66 \\
& TE(s) & 1,96 & 0,70 & 0,48 & 0,60 & 1,22 \\
& TMD(s) & 1,66 & 0,87 & 0,87 & 0,86 & 0,83 \\
Conjunto III & DPRM & 0,14 & 0,08 & 0,03 & 0,05 & 0,12 \\
(11 inst.) & T (s) & 8,68 & 11,16 & 7,90 & 8,95 & 12,09 \\
& TE(s) & 2,88 & 2,97 & 1,94 & 2,59 & 4,34 \\
& TMD(s) & 3,91 & 5,38 & 3,85 & 3,84 & 4,19 \\
Conjunto IV & DPRM & 0,61 & 0,05 & 0,80 & 0,41 & 0,35 \\
(3 inst.) & T (s) & 32,27 & 21,91 & 26,76 & 32,01 & 42,75 \\
& TE(s) & 18,47 & 12,92 & 16,87 & 21,53 & 32,95 \\
& TMD(s) & 7,69 & 7,50 & 7,43 & 8,19 & 8,11 \\
\hline
\end{tabular}


Foram realizados testes adicionais aumentando o tamanho de Refset, mas a melhoria dos resultados é pequena (quando o tamanho da instância é grande) ou não existe (instâncias pequenas), enquanto que o tempo computacional cresce bastante. As soluções obtidas pelo algoritmo da $\mathrm{BD}$ em todas as instâncias quando o tamanho de Refset é 10 e $b_{1}=5$ e $b_{2}=5$ são muito boas em termos de DPRM e tempo computacional, com um desvio percentual menor que $1 \%$ em relação às melhores soluções disponíveis na literatura. Em algumas instâncias foi possível igualar os melhores resultados disponíveis na literatura; em outras poucas instâncias (por exemplo na instância A-n37-k5.vrp de Augerat et alii, 1995) foram obtidas melhores soluções que as reportadas na literatura. É interessante notar que razoável percentagem do tempo computacional, na maioria das instâncias, é consumida ao gerar o conjunto de soluções iniciais (conjunto $P$ ).

Para uma melhor ilustração do comportamento do algoritmo da BD com os diferentes tamanhos de Refset apresentamos nas Figuras 7(a) a 7(d) o diagrama de caixas (ver Montgomery, 2004) para cada conjunto de dados. Os diagramas de caixas descrevem simultaneamente várias características importantes dos resultados obtidos, tais como a mediana (representada pela linha separadora no interior de cada caixa) e a dispersão dos DPR em relação ao DPRM (representada pela amplitude de cada caixa), entre outros. Os diagramas da Figura 7 foram obtidos rodando o Software-R (The R Project for Statistical Computing; para mais detalhes acessar http://www.r-project.org/).

Na Figura 7(a) observamos que para os valores de $b_{1}=5$ e $b_{2}=5$ a maioria dos DPR's são inferiores à mediana, mostrando que o algoritmo de $\mathrm{BD}$, para esses valores, tem um comportamento estável para o Conjunto I. Já quando $b_{1}=3$ e $b_{2}=7$ os resultados relativos aos dados do Conjunto I apresentaram a maior dispersão. A Figura 7(b) mostra que o algoritmo de BD teve o melhor desempenho para Conjunto II quando $b_{1}=7$ e $b_{2}=3$. Na Figura 7(c) o algoritmo da $\mathrm{BD}$, para todos os diferentes valores de $b_{1}$ e $b_{2}$, tem um outlier $(\mathrm{DPR}=-5.24)$, pois em uma instância do Conjunto III foi encontrado um valor da função objetivo menor que o melhor resultado reportado na literatura; observa-se também que quando $b_{1}=3$ e $b_{2}=7$ o algoritmo tem seu melhor comportamento para os dados desse conjunto. Na Figura 7(d) apresenta-se o diagrama de caixas para o Conjunto IV, formado por apenas 3 instâncias.

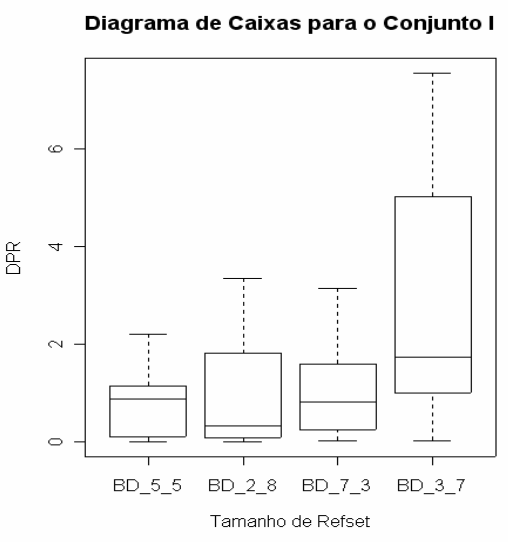

(a)

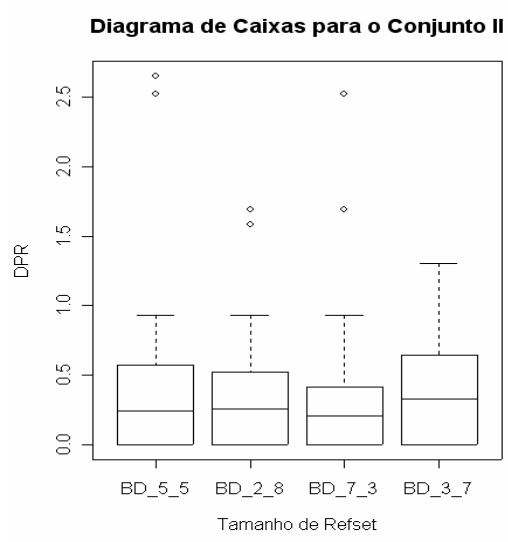

(b) 


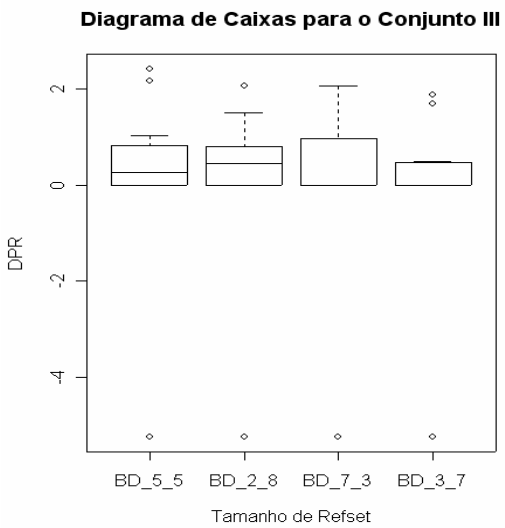

(c)

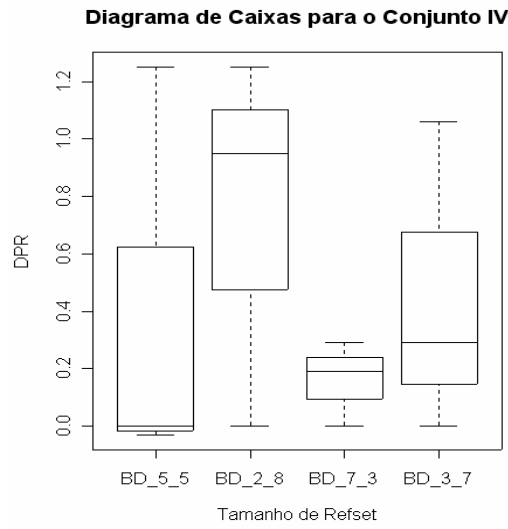

(d)

Figura 7 - Diagramas de Caixas para os Conjuntos Testados.

Se avaliarmos os diagramas da Figura 7 como um todo podemos afirmar que a composição de Refset para $b_{1}=5$ e $b_{2}=5$ tem em média um desempenho estável para todos os conjuntos de dados avaliados.

\section{Conclusões e Perspectivas}

Neste artigo descrevemos um algoritmo de Busca Dispersa desenvolvido para o PRV Clássico, testando seu desempenho para quatro conjuntos de dados disponíveis na literatura. Resultados computacionais mostram que as soluções encontradas pelo algoritmo de BD são bastante próximas às melhores soluções reportadas na literatura para essas instâncias, tendo sido obtidas em tempo computacional reduzido. Resultados ainda melhores poderão ser obtidos com a implementação de recursos avançados da $\mathrm{BD}$, tais como por exemplo sua combinação com outras meta-heurísticas utilizadas com sucesso (e.g., Busca Tabu), para resolver tanto o PRV Clássico como PRV's com restrições adicionais.

\section{Agradecimentos}

Os autores agradecem ao CNPq pelo apoio financeiro recebido, na forma de uma bolsa de doutorado com duração de 4 anos para a autora e de uma bolsa de Pesquisador para o primeiro co-autor. Agradecem também os comentários e sugestões de avaliadores anônimos, que proporcionaram a melhoria do texto.

\section{Referências Bibliográficas}

(1) Augerat, J.R.; Belenguer, J.M. \& Benevant, E. (1995). Computational results with a branch-and-cut code for the capacitated vehicle routing problem. Technical Report RR940-M, University Joseph Fourier, Grenoble.

(2) Baker, M.B. \& Ayechew, M.A. (2003). A genetic algorithm for the vehicle routing problem. Computers and Operations Research, 30, 787-800. 
(3) Ball, M.O.; Magnanti, T.L.; Monma, C.L. \& Nemhauser, G.L. [Editors] (1995). Network Routing. Handbook in Operations Research and Management Science, North Holland.

(4) Beasley, J.E. (1983). Route-first cluster-second methods for vehicle routing. Omega, 11, 403-408.

(5) Campos, V.; Glover, F.; Laguna, M. \& Martí, R. (2001). An experimental evaluation of a scatter search for the linear ordering problem. Journal of Global Optimization, 21, 397-414.

(6) Christofides, N. \& Eilon, S. (1969). An algorithm for the vehicle-dispatching problem. Operational Research Quarterly, 20, 309-318.

(7) Christofides, N.; Mingozzi, A. \& Toth, P. (1979). The vehicle routing problem. In: Combinatorial Optimization [edited by N. Christofides, A. Mingozzi, P. Toth, and C. Sandi], Wiley, Chichester, UK], 315-338.

(8) Cordeau, J-F.; Gendreau, M.; Laporte, G. \& Semet, F. (2002). A guide to vehicle routing problems. Journal of the Operational Research Society, 53, 512-522.

(9) Fisher, M.L. (1994). Optimal solution of vehicle routing problems using minimum K-trees. Operations Research, 42, 626-642.

(10) Gillett, B. \& Miller, L.R. (1974). A heuristic algorithm for the vehicle dispatch problem. Operations Research, 22, 340-349.

(11) Glover, F. (1977). Heuristic for integer programming using surrogate constraints. Decision Sciences, 8, 156-166.

(12) Glover, F. (1997). A template for scatter search and path relinking. In: Lecture Notes in Computer Science [edited by J.K. Hao, E. Lutton, E. Ronald, M. Schoenauer, and D. Snyers], Springer-Verlag, 13-54.

(13) Laguna, M. \& Martí, R. (2000). Experimental testing of advanced scatter search designs for global optimization of multimodal functions. Technical Report TR11-2000, Departamento de Estadística e I.O., Universidad de Valencia.

(14) Laguna, M. \& Martí, R. (2003). Scatter Search Methodology and Implementations in C. Kluwer Academic Publishers.

(15) Laporte, G.; Gendreau, M.; Potvin, J-Y. \& Semet, F. (2000). Classical and modern heuristics for the vehicle routing problem. International Transactions in Operational Research, 7, 285-300.

(16) Lenstra, J. \& Rinnooy, K.A. (1981). Complexity of vehicle routing and scheduling problems. Networks, 11, 221-227.

(17) Lin, S. (1965). Computer solution of the traveling salesman problem. Bell System Technical Journal, 44, 2245-2269.

(18) Martí, R.; Laguna, M. \& Glover, F. (2006). Principles of scatter search. European Journal of Operational Research, 169, 359-372.

(19) Martinhon, C.; Lucena, A. \& Maculan, N. (2004). Stronger K-tree relaxations for the vehicle routing problem. European Journal of Operational Research, 158, 56-71. 
(20) Montané, F.A.T. \& Galvão, R.D. (2006). A tabu search algorithm for the vehicle routing problem with simultaneous pick-up and delivery service. Computers and Operations Research, 33, 595-619.

(21) Montgomery, D.C.; Runger, G.C. \& Hubele, N.F. (2004). Estatística Aplicada à Engenharia. LTC - Livros Técnicos e Científicos Editora S.A., 2.ed., Rio de Janeiro, Brasil.

(22) Russell, R.A. \& Chiang, W. (2006). Scatter search for the vehicle routing problem with time windows. European Journal of Operational Research, 169, 606-622.

(23) Toth, P. \& Vigo, D. (2002). The Vehicle Routing Problem. SIAM Monographs on Discrete Mathematics and Applications, Philadelphia, U.S.A.

(24) Vigo, D. \& Toth, P. (2003). The granular tabu search and its applications to the vehicle routing problem. INFORMS Journal on Computing, 15, 335-346.

(25) Wassan, N.A. (2006). A reactive tabu search for the vehicle routing problem. Journal of the Operational Research Society, 57, 111-116.

\section{APÊNDICE}

Tabela A.1 - Características das instâncias do Conjunto I.

\begin{tabular}{|c|c|c|c|c|c|c|}
\hline Grupo & Instância & $\begin{array}{l}\mathrm{N}^{\circ} \text {. } \\
\text { clientes }\end{array}$ & $\begin{array}{l}\text { Capacidade } \\
\text { do veículo }\end{array}$ & $\begin{array}{l}\text { Tempo de } \\
\text { serviço }\end{array}$ & $\begin{array}{l}\text { Tempo } \\
\text { máximo de } \\
\text { rota }\end{array}$ & $\begin{array}{l}\text { Melhor solução } \\
\text { disponível na } \\
\text { literatura }\end{array}$ \\
\hline \multicolumn{7}{|c|}{ Dados distribuídos aleatoriamente } \\
\hline \multirow{5}{*}{ I } & 1 & 50 & 160 & 0 & $\infty$ & 524,61 \\
\hline & 2 & 75 & 140 & 0 & $\infty$ & 835,26 \\
\hline & 3 & 100 & 200 & 0 & $\infty$ & 826,14 \\
\hline & 4 & 150 & 200 & 0 & $\infty$ & 1028,42 \\
\hline & 5 & 199 & 200 & 0 & $\infty$ & 1291,45 \\
\hline \multirow{5}{*}{ II } & 6 & 50 & 160 & 10 & 200 & 555,43 \\
\hline & 7 & 75 & 140 & 10 & 160 & 909,68 \\
\hline & 8 & 100 & 200 & 10 & 230 & 865,94 \\
\hline & 9 & 150 & 200 & 10 & 200 & 1162,55 \\
\hline & 10 & 199 & 200 & 10 & 200 & 1395,85 \\
\hline \multicolumn{7}{|c|}{ Dados distribuídos em agrupamentos } \\
\hline \multirow[t]{2}{*}{ III } & 11 & 120 & 200 & 0 & $\infty$ & 1042,11 \\
\hline & 12 & 100 & 200 & 0 & $\infty$ & 819,56 \\
\hline \multirow[t]{2}{*}{ IV } & 13 & 120 & 200 & 50 & 720 & 1541,14 \\
\hline & 14 & 100 & 200 & 90 & 1040 & 866,37 \\
\hline
\end{tabular}


Tabela A.2 - Características das instâncias do Conjunto II.

\begin{tabular}{|c|c|c|c|c|c|c|}
\hline Instâncias & $\begin{array}{l}\mathrm{N}^{\circ} \text {. } \\
\text { clientes }\end{array}$ & Veículos & $\begin{array}{l}\text { Capacidade } \\
\text { do veículo }\end{array}$ & $\begin{array}{l}\text { Tempo de } \\
\text { serviço }\end{array}$ & $\begin{array}{l}\text { Tempo } \\
\text { máximo de } \\
\text { rota }\end{array}$ & Ótimo \\
\hline A-n32-k5.vrp & 31 & 5 & 100 & 0 & $\infty$ & 784 \\
\hline A-n33-k5.vrp & 32 & 5 & 100 & 0 & $\infty$ & 661 \\
\hline A-n33-k6.vrp & 32 & 6 & 100 & 0 & $\infty$ & 742 \\
\hline A-n34-k5.vrp & 33 & 5 & 100 & 0 & $\infty$ & 778 \\
\hline A-n36-k5.vrp & 35 & 5 & 100 & 0 & $\infty$ & 799 \\
\hline A-n37-k5.vrp & 36 & 5 & 100 & 0 & $\infty$ & 669 \\
\hline A-n37-k6.vrp & 36 & 6 & 100 & 0 & $\infty$ & 949 \\
\hline A-n38-k5.vrp & 37 & 5 & 100 & 0 & $\infty$ & 730 \\
\hline A-n39-k5.vrp & 38 & 5 & 100 & 0 & $\infty$ & 822 \\
\hline A-n39-k6.vrp & 38 & 6 & 100 & 0 & $\infty$ & 831 \\
\hline A-n44-k6.vrp & 43 & 6 & 100 & 0 & $\infty$ & 937 \\
\hline A-n45-k6.vrp & 44 & 6 & 100 & 0 & $\infty$ & 944 \\
\hline A-n45-k7.vrp & 44 & 7 & 100 & 0 & $\infty$ & 1146 \\
\hline A-n46-k7.vrp & 45 & 7 & 100 & 0 & $\infty$ & 914 \\
\hline A-n48-k7.vrp & 47 & 7 & 100 & 0 & $\infty$ & 1073 \\
\hline A-n53-k7.vrp & 52 & 7 & 100 & 0 & $\infty$ & 1010 \\
\hline A-n54-k7.vrp & 53 & 7 & 100 & 0 & $\infty$ & 1167 \\
\hline A-n55-k9.vrp & 54 & 9 & 100 & 0 & $\infty$ & 1073 \\
\hline A-n60-k9.vrp & 59 & 9 & 100 & 0 & $\infty$ & 1354 \\
\hline A-n61-k9.vrp & 60 & 9 & 100 & 0 & $\infty$ & 1034 \\
\hline A-n62-k8.vrp & 61 & 8 & 100 & 0 & $\infty$ & 1288 \\
\hline A-n63-k10.vrp & 62 & 10 & 100 & 0 & $\infty$ & 1616 \\
\hline A-n63-k9.vrp & 62 & 9 & 100 & 0 & $\infty$ & 1314 \\
\hline A-n64-k9.vrp & 63 & 9 & 100 & 0 & $\infty$ & 1401 \\
\hline A-n65-k9.vrp & 64 & 9 & 100 & 0 & $\infty$ & 1174 \\
\hline A-n69-k9.vrp & 68 & 9 & 100 & 0 & $\infty$ & 1159 \\
\hline A-n80-k10.vrp & 79 & 10 & 100 & 0 & $\infty$ & 1763 \\
\hline
\end{tabular}

Tabela A.3 - Características das instâncias do Conjunto III.

\begin{tabular}{|c|c|c|c|c|c|c|}
\hline Instância & $\begin{array}{l}\mathbf{N}^{\circ} . \\
\text { clientes }\end{array}$ & Veículos & $\begin{array}{l}\text { Capacidade } \\
\text { do veículo }\end{array}$ & $\begin{array}{l}\text { Tempo de } \\
\text { serviço }\end{array}$ & $\begin{array}{l}\text { Tempo } \\
\text { máximo de } \\
\text { rota }\end{array}$ & Ótimo \\
\hline E-n22-k4.vrp & 21 & 4 & 6000 & 0 & $\infty$ & 375 \\
\hline E-n23-k3.vrp & 22 & 3 & 4500 & 0 & $\infty$ & 569 \\
\hline E-n30-k3.vrp & 29 & 3 & 4500 & 0 & $\infty$ & 534 \\
\hline E-n33-k4.vrp & 32 & 4 & 8000 & 0 & $\infty$ & 835 \\
\hline E-n51-k5.vrp & 50 & 5 & 160 & 0 & $\infty$ & 521 \\
\hline E-n76-k7.vrp & 75 & 7 & 220 & 0 & $\infty$ & 682 \\
\hline E-n76-k8.vrp & 75 & 8 & 180 & 0 & $\infty$ & 735 \\
\hline E-n76-k10.vrp & 75 & 10 & 140 & 0 & $\infty$ & 830 \\
\hline E-n76-k14.vrp & 75 & 14 & 100 & 0 & $\infty$ & 1021 \\
\hline E-n101-k8.vrp & 100 & 8 & 200 & 0 & $\infty$ & 815 \\
\hline E-n101-k4.vrp & 100 & 14 & 112 & 0 & $\infty$ & 1067 \\
\hline
\end{tabular}


Tabela A.4. Características das instâncias do Conjunto IV.

\begin{tabular}{l|l|l|l|l|l|r}
\hline Instância & \multicolumn{1}{l|l}{$\begin{array}{l}\text { No. } \\
\text { clientes }\end{array}$} & Veículos & $\begin{array}{l}\text { Capacidade } \\
\text { do veículo }\end{array}$ & $\begin{array}{l}\text { Tempo de } \\
\text { serviço }\end{array}$ & $\begin{array}{l}\text { Tempo } \\
\text { máximo de } \\
\text { rota }\end{array}$ & Ótimo \\
\hline F-n45-k4.vrp & 44 & 4 & 2010 & 0 & $\infty$ & 723,54 \\
F-n72-k4.vrp & 71 & 4 & 30000 & 0 & $\infty$ & 241,97 \\
F-n135-k7.vrp & 134 & 7 & 2210 & 0 & $\infty$ & 1163,60 \\
\hline
\end{tabular}

